\title{
La reconstrucción organizativa del pueblo guaraní en Bolivia y sus acciones colectivas por el territorio
}

Organizational reconstruction of the Guarani people in Bolivia and their collective actions for territory

\begin{tabular}{ll}
\hline Nombre: & Juan Wahren $\left(^{1}\right)$ \\
Filiación: & Universidad de Buenos Aires \\
País: & Argentina \\
Correo: & juanwahren@yahoo.com.ar
\end{tabular}

\section{RESUMEN}

En este trabajo nos proponemos dar cuenta de las transformaciones organizativas del pueblo guaraní de Bolivia a partir del análisis de las acciones colectivas de la Asamblea del Pueblo Guaraní (APG) en torno al territorio, los recursos naturales y la recuperación cultural e identitaria. Esta organización surgióa fines de la década del ochenta en la región del sureste boliviano, una zona muy rica en recursos naturales.Asimismo, este movimientoha conformado un amplio marco de alianzas con otras organizaciones indígenas y campesinas de Bolivia.En este marco, se analizan también algunas de las tensiones existentes entre este proceso de acción colectiva y reconfiguración identitaria de los movimientos sociales y las políticas del Estado boliviano en torno a los recursos naturales y las demandas indígenas sobre las cuestiones culturales e identitaria. La metodología planteada en el artículo es fundamentalmenteuna estrategia cualitativa basada en entrevistas y observaciones de campo.

\section{PALABRAS CLAVE}

Identidad, pueblos indígenas, movimientos sociales, acciones colectivas, territorialidad, Bolivia.

${ }^{1}$ El autor es Doctor en Ciencias Sociales y Becario Postdoctoral del CONICET. Es parte del Grupo de Estudios de los Movimientos Sociales de América Latina (GEMSAL) del Instituto de Investigaciones Gino Germani (IIGG) en la Facultad de Ciencias Sociales (FCS), Universidad de Buenos Aires (UBA). Sus líneas de investigación son: movimientos sociales, territorialidad, cambio climático y sociología rural. 


\section{ABSTRACT}

This article proposes to account for the organizational changes of the Guarani indigenous people from Bolivia analyzing the collective actions of the Asamblea del Pueblo Guaraní (APG) -the Guaraní People Assembly- regarding territory, natural resources, cultural recovery and indigenous identity. This organization begins in the end of the 80 's in the Southeast of Bolivia, an area rich in natural resources. Also, this movement has formed a broad framework of alliances with other indigenous and peasant organizations in Bolivia. In this context, we analyzes some of the tensions between processes of collective action, identity reconfiguration of social movements and the Bolivian State policies regarding natural resources and indigenous demands for cultural and identity issues. The methodology proposed in this paper is qualitative, based on interviews and field observations.

\section{KEY WORDS}

Identity, Indigenous People, Social Movements, collective Actions, Territoriality, Bolivia.

\section{Introducción}

En el marco del avance del neoliberalismo y la profunda crisis del Estado en Bolivia durante las décadas del ochenta y noventa, los movimientos sociales bolivianos sufrieron importantes cambios políticos, culturales y económicos que afectaron a sus organizaciones e incluso transformaron en parte sus propias identidades políticas y sociales. En este contexto, se produce un resurgimiento de las identidades indígenas a partir de la pérdida de la centralidad del movimiento obrero, particularmente los trabajadores mineros, que habían sido el actor hegemónico junto -y a veces en tensión- con las organizaciones campesinas-indígenas del conflicto social boliviano en las décadas anteriores. En este trabajo nos focalizamos en el proceso de recuperación cultural e identitaria del pueblo guaraní, así como del surgimiento de la Asamblea del Pueblo Guaraní (APG) como instrumento organizativo de este pueblo originario de las "tierras bajas" del oriente boliviano. Analizamos la interrelación entre los procesos organizativos e identitarios del pueblo guaraní en el contexto de la reconfiguración identitaria de los pueblos indígenas de Bolivia en las últimas décadas del siglo XX, así como las diferentes acciones colectivas de protesta en torno al territorio y los recursos naturales.

La APG surge en el año 1987 integrando a más de 80.000 guaraní en su seno. Es una organización de segundo grado que aglutina a distintas comunidades guaraníes del sureste boliviano, en los departamentos de Chuquisaca, Tarija y Santa Cruz. Las comunidades, ubicadas en zonas rurales, constituyen los núcleos de base de la 
organización. Estas comunidades se agrupan a su vez en distintas capitanías cuyo responsable es un Capitán Grande -MburuvichaGuasu en guaraní-, que se rige de acuerdo a los usos y costumbres del pueblo guaraní con la incorporación de ciertas lógicas organizativas sindicales.A su vez la APG se encuentra dentro de la Confederación de Pueblos indígenas del Oriente Boliviano (CIDOB), que es una organización nacional que agrupa a los diferentes pueblos indígenas del oriente boliviano (de los departamentos de Santa Cruz, Pando, Beni y parte de Chuquisaca), y es una de las organizaciones indígenas más importantes de Bolivia, con un fuerte protagonismo en las acciones colectivas de protesta que se desencadenaron desde mediados de la década del noventa hasta la actualidad en ese país. La APG, junto con otros movimientos campesinos, indígenas y vecinales fueron los que posibilitaron, directa o indirectamente, el triunfo electoral del Movimiento al Socialismo (MAS), estructura política que canalizó las demandas políticas de varios de los movimientos sociales bolivianos. Con la llegada del líder cocalero Evo Morales al Gobierno Nacional y el llamado a una nueva Asamblea Constituyente a fines de 2005, se cerró el "ciclo de protesta"(Tarrow, 2009) iniciado cinco años antes durante la llamada "Guerra del Agua" de Cochabamba y continuado con la denominada "Guerra del Gas" de 2003 (Svampa et al., 2010). Sin embargo, a partir de 2010 emergieron nuevos conflictos protagonizados por diferentes movimientos sociales -donde la APG tiene un protagonismo importante- ligados a disputas por territorios y recursos naturales que permiten presuponer la apertura de un nuevo ciclo de protesta en una compleja relación con el nuevo Estado plurinacional boliviano.

En este sentido, la APG protagonizó en los últimos años un importante conflicto en la región de Campo Margarita (en el departamento de Tarija) donde la empresa multinacional de hidrocarburos REPSOL YPF gestiona uno de los yacimientos hidrocarburíferos más grandes de Bolivia. Este conflicto, ligado a los diferentes usos y sentidos otorgados a los recursos naturales en esos territorios habitados tradicionalmente por las comunidades guaraní, quedó en un "impasse” entre el año 2006 y el 2009 cuando se reactivó el conflicto. Aún más, desde finales de ese mismo año, estas disputas territoriales se han ampliado hacia otras zonas de la región chaqueña del departamento de Tarija en la provincia del Gran Chaco compuesta por Yacuiba, Karaparí y Villa Montes, donde la APG tiene organizaciones de base y zonales por cada municipio.

En este trabajo utilizamos diversas fuentes secundarias para realizar la reconstrucción histórica e identitaria del Pueblo Guaraní,contando para ello con bibliografía académica especializada que da cuenta del proceso organizativo y la historia del pueblo guaraní, principalmente enfocados en las transformaciones recientes del pueblo guaraní a partir de la conformación de la Asamblea del Pueblo Guaraní a fines de la década del ochenta. Para el análisis de la etapa actual de las acciones colectivas de la APG utilizamos, también, fuentes primarias obtenidas en el marco de trabajos de campo realizados durante los años 2009 y 2010 en Tarija. En este contexto, se realizaron entrevistas a dirigentes de los distintos niveles organizativos de la APG y a diferentes miembros "de base" y "técnicos" de la 
organización, así como se realizaron observaciones de reuniones, asambleas y movilizaciones del Pueblo Guaraní durante este período.

\section{Los ciclos de protesta y el rol de la APG (1990-2011)}

En los últimos veinte años podemos vislumbrar en Bolivia tres grandes ciclos de protesta y movilización popular hasta nuestros días: (1) El surgimiento de viejos/nuevos actores sociales entre 1990 y el año 2000,(2) las resistencias insurreccionales al neoliberalismo entre el año 2000 y el 2005, y (3) las acciones colectivas en el marco del "gobierno de los movimientos sociales" desde el año 2006 hasta la actualidad.

Las características generales de estos tres ciclos de protesta pueden resumirse en:(a) la profunda crisis de representación de los partidos políticos tradicionales y del propio andamiaje político-institucional de Bolivia, con el correspondiente "hastío social" frente a la incapacidad de los partidos políticos de solucionar los problemas cotidianos de la población; (b) la capacidad de los movimientos sociales de articular prácticas y discursos, ligados a soluciones concretas para los problemas cotidianos de las poblaciones urbanas y rurales a partir de los propios anclajes identitarios y culturales de los pueblos que existen de manera yuxtapuesta y abigarrada en Bolivia,y que implican la creación de nuevas formas y lógicas de lo político "más allá" de lo institucional; y (c) una resignificación de las relaciones entre los movimientos sociales y el Estado en el marco de un "proceso de articulación de alianzas entre diversos sectores de la sociedad" para la creación de nuevos espacios de participación política directa y autónoma en relación a cambios radicales en las normas institucionales que reconozcan e incluso promuevan estas nuevas formas de participación política (Chávez et al., 2010). A estas características planteadas por estas autoras creemos importante incorporar la dimensión de la “territorialidad”(Porto Goncalves, 2002;MancanoFernandes, 2005) como un componente que atraviesa de manera transversal las acciones colectivas de protesta de los movimientos sociales.

En el primer ciclo de protesta aparecen nuevas demandas, con acciones colectivas resignificadas a partir del resurgimiento de estos viejos/nuevos actores sociales: los campesinos y los pueblos indígenas. La acción de protesta que inaugura este ciclo es la "Marcha por la Dignidad y el Territorio", que protagonizaronen 1990 los pueblos indígenas de tierras bajas que irrumpen en el escenario político boliviano luego de décadas de silenciamiento e invisibilización. Las demandas estaban vinculadas a exigencias en torno a la distribución de la tierra pero también a los modos de habitarla, es decir, se comenzó a plantear la demanda de territorio como una forma integral de recuperar la tierra, pero también una forma de producir una forma de gobernarse, una forma de reproducción de la vida. Así, para la cosmovisión indígenael territorio representa "un espacio de vida esencial para la reproducción social, cultural, religiosa, económica y política. El territorio es integral, una unidad 
que no se presta a división porque implica la pluralidad de recursos que lo pueblan, sea el suelo, el subsuelo o el aire; es la base de la subsistencia, un espacio vital multidimensional y no un bien comercial" (Fornillo, 2010:177). Las otras demandas se encontraban ligadas al reconocimiento de la cultura y de la existencia política de los pueblos indígenas de Bolivia.

En este marco general del "ciclo de protesta del resurgimiento de los viejos/nuevos actores sociales", se da la conformación de la APG, fundada en 1987 como culminación de un largo proceso de reconstrucción de las redes organizativas del pueblo Guaraní (García Linera et al., 2008;Orozco Ramírez et al., 2006) que había sido casidesarticulado tanto por el sistema de haciendas de la región como por los procesos migratorios de los mundos rurales hacia las ciudades que marcó gran parte del siglo XX, así como por la creciente dificultad del acceso a la tierra para el pueblo guaraní $\mathrm{y}$, más recientemente, el arrinconamiento de las comunidades indígenas por parte de empresas petroleras $\mathrm{y} / \mathrm{o}$ agroganaderas $\mathrm{y}$ forestales. $\mathrm{El}$ proceso organizativo de los guaraní implicó una "combinación de tradición y modernidad" (Bazoberry y Heredia, 2004:162), donde aparecen reconfigurados roles tradicionales de la organización del pueblo guaraní con formas organizativas ligadas a las tradiciones sindicales y campesinas del siglo XX de Bolivia que fueron acercadas por técnicos de distintas ONG's, sectores de la Iglesia católica comprometidos con los pueblos indígenas en las tierras bajas y activistas de distintos agrupamientos sindicales y políticos a lo largo de la segunda mitad del siglo XX.

En su inicio la lucha por la tierra está muy ligada a lucha de las comunidades por su independencia. Estamos hablando de la década de los 80, imagínate, todavía de comunidades que eran cautivas en haciendas, entonces el hacendado tenía sus tierras y dentro de ellas había comunidades, se vendían las tierras y se vendían las comunidades. Entonces, más que una noción territorial, en ese primer momento, el disparador es la independencia, es el "queremos trabajar para nosotros", ese es un poco el discurso de los guaraníes (Entrevista a Miguel Castro, AVINA - Bolivia, ex Presidente del CERDET, 2009).

El surgimiento de la APG se da inicialmente en el departamento de Santa Cruz y en menor medida en el de Chuquisaca, particularmente en la región de Tentayapi. En la década del ochenta, la mayoría de los guaraní de Tarija se encontraban dispersos, sin organización comunitaria de base 0 en situación de "empatronamiento".

Una vez que salía de la hacienda, la gente era “libre” pero no sabía cómo gestionar su vida porque el sistema de hacienda había sido muy duro, no 
había un guaraní que conozca todo el proceso productivo del maíz porque ellos participaban en pedazos. El reto era casi de descolonización, o sea, cómo romper el sistema de la hacienda en el imaginario indígena. $Y$ ahí es cuando surge por primera vez las palabras “tierra”, “territorio", como la necesidad de hacer sostenible esa libertad, es decir, ya no estamos en la hacienda pero de qué vamos a vivir. Incluso en esa época muchos guaranís quieren volver a la hacienda porque de alguna manera les daba seguridad. Entonces dispara con mucha fuerza la demanda territorial (Entrevista a Miguel Castro, AVINA - Bolivia, ex Presidente del CERDET, 2009).

Lo mismo sucedía en distintas zonas de Santa Cruz hacia donde se expandió el proceso organizativo de la APG en sus comienzos. La APG comienza su trabajo en Tarija a principios de los años noventa, principalmente en la región del Itika Guasu. Los guaraní que habitan las zonas rurales se encuentran organizados por comunidades de base que en grupos de entre 10 y 30 conforman capitanías regionales. Estas capitanías regionales se agrupan y conforman, a su vez, capitanías generales por departamento. Sobre esa estructura de organización tradicional que se fue recuperando a partir de los años setenta se conformó la estructura organizativa de lo que hoy es la Asamblea del Pueblo Guaraní (APG) en Tarija, que junto con la Asamblea de los departamentos de Santa Cruz y Chuquisaca conforman la APG a nivel nacional. La de Tarija está conformada actualmente por seis capitanías zonales: Yacuiba, Villa Montes y Karaparí con 57 comunidades organizadas en la provincia del Gran Chaco y las tres capitanías del ItikaGuasu (Zona I, Zona II y Zona III) en la provincia de O'Connor, con 36 comunidades en total. En la zona de Bermejo existen algunas comunidades guaraní en un incipiente proceso organizativo apoyado desde la APG de Tarija. Esto da un total de aproximadamente 95 comunidades organizadas en la APG de Tarija².

De esta manera, la APG a nivel nacional articula entonces a las comunidades guaraní más allá de las fronteras departamentales que el Estado nación republicano impuso hacia finales del siglo XIX y recupera, en parte, la movilidad y articulación política y cultural histórica del pueblo guaraní. Pocos años después del proceso fundacional de la APG comienzan las primeras acciones colectivas de protesta de los pueblos indígenas de tierras bajas donde participa la APG. Las demandas giraban en un principio en torno al acceso a la tierra; paulatinamente esta demanda se fue expandiendo hacia los usos y sentidos de los territorios indígenas y sobre los recursos naturales que allí se encuentran, en consonancia con las disputas planteadas por el

\footnotetext{
${ }^{2}$ En el ItikaGuasu viven 818 familias en 36 comunidades. En la zona Karaparí viven 230 familias en 17 comunidades, en la zona Yacuiba viven 650 familias en 17 comunidades y en la zona Villa Montes viven 523 familias en 13 comunidades. A esto hay que sumarle aproximadamente media docena de comunidades en la provincia de Bermejo que se encuentran en un proceso de recuperación identitaria, cultural y organizativa.
} 
conjunto de los movimientos indígenas bolivianos que ampliaron sus demandas más allá del acceso a la tierra, insistiendo por el control y gestión plena de los territorios ancestrales y los recursos naturales que allí se encuentran.

Algunas de estas acciones colectivas de los movimientos indígenas de Bolivia muchas de las cuales contaron con la activa participación de la APG- fueron las movilizaciones de 1996, 2000 y $2002^{3}$ de los pueblos de las tierras bajas y las marchas cocaleras iniciadas en 1994 en defensa del cultivo y la comercialización tradicional de la coca marcaron una continuidad de las acciones colectivas y demandas iniciadas a comienzos de la década del noventa con la paulatina incorporación a las movilizaciones de sectores populares del occidente, principalmente las organizaciones aymaras del sector rural como la CONAMAQ y del sector urbano como las comisiones vecinales de la ciudad de El Alto. El principal repertorio de acción de este ciclo fueron lo que aquí denominamos la "marcha larga", que implica una movilización que recorre grandes distancias -cientos de kilómetros- desde comunidades remotas hacia el centro político de la república (la ciudad de La Paz) o alguna otra ciudad de importancia política y/o económica a nivel departamental o regional. Este tipo de movilizaciones no estuvieron exentas de cruentas represiones y enfrentamientos con la policía y otras fuerzas de seguridad, pero aseguraban una importante cobertura mediática a los reclamos y en general generaban una amplia solidaridad de otros sectores populares y clases medias urbanas. En los finales de este ciclo de protesta comenzó a esbozarse la demanda por una Asamblea Constituyente, reclamo que se cristalizó en las jornadas de la llamada "Guerra del Agua" en Cochabamba en el año 2000, que dio inicio al segundo ciclo de protesta.

Este segundo ciclo de protesta -el "ciclo insurreccional"-se vio signado por la incorporación plena a las protestas sociales de los sectores populares del occidente. Por un lado, la confederación campesina (CSUTCB) conducida por Felipe Quispe de orientación "katarista", por otro lado la consolidación de las organizaciones regionales indígenas quechuas y principalmente aymaras cuya organización principal es la CONAMAQ (García Linera et al., 2008), ylos sindicatos cocaleros consolidados bajo elliderazgo de Evo Morales Ayma de orientación más “clasista”, pero con cada vez mayores componentes identitarios indígenas a partir de la luchas por la defensa del uso y comercialización de la coca como "cultivo ancestral"(Stefanoni, 2003; García Linera et al., 2008),y finalmente la irrupción del complejo entramado social

${ }^{3}$ Esta movilización del año 2002 -la "Marcha por la Asamblea Constituyente, por la Soberanía Popular, el Territorio y los Recursos Naturales"- fue una de las más masivas de los pueblos de indígenas de las tierras bajas.De hecho, aparece como una acción colectiva bisagra entre los dos ciclos de protesta, pues si bien mantiene el formato de movilización de comunidades enteras desde zonas remotas hacia el centro político del país, recoge la nueva demanda de Asamblea Constituyente y logra articular en la práctica a organizaciones indígenas del oriente con organizaciones de pueblos indígenas altiplánicos, principalmente aymaras integrantes de la CONAMAQ. Para un relato pormenorizado de esta movilización y su influencia en la posterior convocatoria a una Asamblea Constituyente, ver el trabajo de Romero Bonifaz (2005). 
que dio lugar a la "Guerra del Agua" en Cochabamba entre sectores indígenas, campesinos, sectores medios urbanos, trabajadores industriales, docentes, profesionales, estudiantes, etc., que lograron frenar el proyecto privatizador del sistema de riego y agua de la ciudad de Cochabamba y el sector rural adyacente. Ello dejó como saldo organizativo a la Coordinadora en Defensa del Agua y la Vida, a la vez que un aprendizaje insurreccional en todo el movimiento social boliviano (Ceceña, 2005; García Linera et al., 2008).

A partir de este acontecimiento se logró, por un lado, derrotar un proyecto privatizador de la segunda oleada neoliberal en Bolivia; por otro lado, permitió articular de manera exitosa a un conjunto de organizaciones y actores sociales que hasta entonces venían protagonizando protestas y acciones colectivas de manera dispersa y con una débil articulación más cercana a la solidaridad que a la puesta en común de acciones concretas y demandas unificadas (Ceceña, 2005).

Además de la demanda contra la privatización de los recursos naturales -en este caso el agua-, se comenzó a articular un discurso acerca de la soberanía popular en torno a los territorios y los recursos naturales que influirá de manera contundente en las protestas venideras en torno a los hidrocarburos. Al mismo tiempo, en estas protestas se retomó y nacionalizó la demanda por una Asamblea Constituyente que diera cuenta de una nueva forma de relación entre gobernantes y gobernados, en un país donde estallaban los mecanismos de dominación coloniales que habían supuesto la construcción de una nación mestiza que se resquebrajaba rápidamente con la emergencia de estos nuevos/viejos actores -los indígenas y campesinos- que venían a señalar la inviabilidad de un Estado nación que negara a la mayoría de su población, perteneciente a los múltiples y diversos pueblos y culturas que existían en el subsuelo boliviano de manera soterrada, yuxtapuesta, "abigarrada" (Zavaleta Mercado, 2008).

Masivas movilizaciones, cortes de rutas, huelgas de hambre, barricadas y enfrentamientos callejeros con la policía y el ejército boliviano fueron parte del repertorio de acciones que desplegó el conjunto de actores involucrados en las luchas que conformaron este segundo ciclo de protesta. Protestas protagonizadas por viejos/nuevos actores sociales que ahora (re)cobraban su voz y su lugar en la política nacional desde una práctica y un discurso radical en las calles, en las rutas, en las quebradas, valles y montañas de una Bolivia sorprendida en pleno auge neoliberal.

Otra gran insurrección popular de este ciclo se produjo en el llamado "Febrero Negro" en el año 2003, cuando diversos actores sociales-incluida la policía que tuvo importantes enfrentamientos armados con el ejército en la zona céntrica de La Paz- se rebelaron en contra de las políticas de ajuste económico del gobierno de Sánchez de Losada, propugnado a través de un "impuestazo" a los salarios de los trabajadores bolivianos. En los enfrentamientos callejeros entre manifestantes y el ejército, los primeros quemaron y saquearon diversos edificios públicos identificados con la "clase política" que se encontraba totalmente deslegitimada. 
En Octubre de ese mismo año se desarrolló el mayor conflicto de este ciclo de protesta, tanto por la magnitud de los enfrentamientos como por el alcance nacional de las protestas y sus consecuencias políticas. Estas jornadas se originaron como protesta frente a la decisión del gobierno de exportar gas a través de puertos de Chile. Así se amalgamaron sentimientos nacionalistas -Chile en la Guerra del Pacífico en el año 1879 arrebató a Bolivia la salida al Océano Pacífico- con la cuestión de los hidrocarburos que se encontraba ligada históricamente al sentimiento popular de soberanía nacional luego de la Guerra del Chaco. Frente a la negativa gubernamental de retroceder en esta decisión, las movilizaciones populares se fueron incrementando, abarcando en primer lugar las ciudades de La Paz y el Alto, luego el resto de los departamentos altiplánicos para alcanzar en pocas semanas la totalidad del país movilizado bajo la consigna de frenar la exportación de gas vía Chile, pero sumando las demandas de nacionalización de los hidrocarburos y el llamado a una Asamblea Constituyente. De esta manera se conformaba la denominada "Agenda de los Movimientos Sociales" que marcaría la política boliviana por los siguientes años hasta la actualidad. En el marco de estos acontecimientos, el presidente Sánchez de Losada presentó su renuncia, asumiendo su Vicepresidente Carlos Mesa, quien prometió llamar a un referéndum en torno a esta problemática para avanzar en una nueva Ley de Hidrocarburos. Durante los años 2004 y 2005 continuaron las protestas sociales por la nacionalización de los hidrocarburos. La nueva Ley de Hidrocarburos surgida del referéndum de 2004 y propulsada por Carlos Mesa no contemplaba grandes cambios en la propiedad de los hidrocarburos aunque aumentaba -en partela apropiación de la renta petrolera por parte del Estado.

Durante este segundo ciclo de protesta, la Asamblea del Pueblo Guaraní se destacó por la realización de cortes de acceso en algunas de las empresas multinacionales de hidrocarburos así como en bloqueos de rutas y puentes estratégicos para la producción de petróleo y gas.Así, en articulación con otras organizaciones campesinas e indígenas de las "tierras bajas" del oriente boliviano, la APG fue asumiendo demandas de carácter nacional, como la exigencia de una Asamblea Constituyente y la nacionalización de los hidrocarburos. Paralelamente, comienzan las denuncias contra los casos de contaminación que comenzó a producir la industria hidrocarburíferas en la zona y comenzaron las disputas específicas con algunas empresas, cuyo conflicto paradigmático fue en el Itika Guasu con Repsol YPF.

La estrategia de acción era de bloqueo de rutas, de empresas. Ante un Estado que estaba totalmente a favor de la inversión privada, las organizaciones indígenas tuvieron que tomar por su propia cuenta la lucha por sus reivindicaciones para lograr por lo menos un mínimo en compensaciones por todo el impacto ambiental negativo que estaban recibiendo. Ahí la única salida es la presión, la movilización. Y eso ha 
generado, creo yo, uno de los factores que ha acumulado toda la emergencia popular para que se desencadene la guerra del gas en 2003. Y todas las movilizaciones posteriores también la APG ha sido protagonista, para que se cambie la ley de los hidrocarburos de 2005 (Entrevista a Erick Aráoz, Equipo Técnico CCGTT de la APG Tarija, 2009).

Paralelamente a estas acciones, la APG fue consolidándose territorialmente, expandiendo sus áreas de influencia y organización en Tarija y otros departamento como Santa Cruz y Chuquisaca. El segundo ciclo de protesta se clausura a fines del año 2005 con el triunfo electoral del candidato del Movimiento Al Socialismo (MAS), Evo Morales, quien encarnaba como dirigente histórico de los campesinos "cocaleros" a los movimientos sociales que habían protagonizado los ciclos de protesta desde principios de la década del noventa.

Los primeros años del gobierno del MAS fueron signados por enfrentamientos con los sectores dominantes de los departamentos del oriente boliviano, la denominada "Medialuna" que se oponían a algunas de las medidas más radicalizadas del gobierno de Evo Morales, como la reforma constitucional, la reforma agraria, la nacionalización de los hidrocarburos, entre otras medidas. Recién en el año 2009 comienza a emerger una creciente tensión entre el gobierno "masista" y los movimientos sociales en torno a las disputas por los territorios y los recursos naturales.Es en el año 2010 cuando se retoman algunas acciones colectivas de protesta -la primera de ellas protagonizadas por la APG- que rememoran el repertorio de acciones del primer ciclo de protesta durante la década del noventa: las "marchas largas", es decir, las movilizaciones que recorren decenas o centenas de kilómetros para llamar la atención sobre una determinada problemática, generalmente ligada a los territorios indígenas. Este tipo de acciones suelen tener un fuerte impacto en la opinión pública y en los medios de comunicación que suelen cubrirlas durante cada día de movilización, llegando muchas veces a las portadas de los periódicos regionales y de la prensa de alcance nacional. Así se desarrollaron dos importantes movilizaciones durante el 2010, la primera fue la "Marcha por la defensa del territorio ancestral y el respeto a los derechos indígenas"convocada por la APG de Tarija, más precisamente por las Capitanías de Yacuiba, Villa Montes y Karaparí, cuyas consignas principales eran: “Déjennos aire, tierra y agua, déjennos árboles y animales, déjennos vida"; "La tierra no nos pertenece, porque nosotros somos parte de la tierra" y "Bolivia Yyambae ${ }^{4}$, sin dueños ni patrones". Esta fue la primera movilización de organizaciones indígenas de "tierras bajas" reclamando contra el gobierno de Evo Morales.

Nosotros como consejo de capitanes estamos convocando a una gran marcha. La primer marcha en el departamento de Tarija que estamos convocando

${ }^{4}$ Yyambae significa "ser libre" u "hombre libre" en guaraní. 
para decirle al gobierno, y no solamente al gobierno sino a toda la gente de los municipios, todo los funcionarios, o sea todo los funcionarios públicos, gobierno nacional, departamental, regional, provincial, que respete a los pueblos indígenas, la herencia, ¿no? Que se está perdiendo y en la misma Constitución dice que hay que respetar y proteger los derechos de los pueblos indígenas (Entrevista a Justino Zambrana, presidente del CCGTT de la APG Tarija, 2010).

La segunda movilización de indígenas de “tierras bajas" en el año 2010 fue convocada por la CIDOB y abarcó a gran parte de las organizaciones indígenas de las tierras bajas. La APG a nivel nacional no se plegó a esta movilización, pues obtuvo canales de negociación directos con ministros encargados de las temáticas de autonomía, medio ambiente, hidrocarburos y tierras, con quienes se reunieron en la ciudad de Camiri (Santa Cruz) semanas previas a la movilización anunciada por la CIDOB. Sin embargo, la APG de Tarija decidió participar igualmente de la movilización sin el aval de su organización a nivel nacional.

El propósito de retomar estos ciclos de protesta de los movimientos sociales bolivianos, destacando el rol que tuvo la APG en cada uno de ellos, permite observar cómo las acciones colectivas del pueblo guaraní en disputa por sus territorios y los recursos naturales habilitaron un proceso de reconfiguración identitaria y cultural como pueblo. En este sentido, "la reestructuración del pueblo guaraní ha significado el desarrollo de un discurso donde se construye una noción de territorio, después de uso y aprovechamiento de recursos, para pasar al campo político, donde la cultura y la identidad se han convertido en reivindicación política" (Bazoberry y Heredia, 2004:166). Así, el pueblo guaraní aparece en el escenario político boliviano durante la década del noventa y continúa gravitando con proyección regional y nacional en las discusiones en torno a la autonomía, los recursos naturales, la tierra y el territorio, la cuestión étnica y cultural, así como en la discusión de un Estado plurinacional al mismo nivel que los pueblos indígenas quechuas y aymaras, y otros movimientos sociales bolivianos. Es en este sentido que ligamos las disputas de los guaraní por el reconocimiento cultural como pueblo y de los territorios que habitan como una posibilidad de obtener una autonomía territorial y política, como proyecto emancipatorio que va más allá de las autonomías municipales, regionales y departamentales planteadas en el marco institucional de las distintas formas de autonomía reconocidas en la nueva constitución política de Bolivia.

Es recién durante el segundo mandato de Evo Morales (fue reelecto en diciembre del año 2009 con el $64,2 \%$ de los votos) cuando comenzaron a sentirse con mayor fuerza algunas de las tensiones entre el gobierno y los movimientos sociales en torno a varias demandas pendientes, o frente al propio accionar de la política gubernamental. En este sentido, continúa abierto el debate del rol del nuevo "Estado plurinacional" en torno al creciente avance de los emprendimientos 
hidrocarburíferos en los territorios campesinos e indígenas, sobre todo en la región chaqueña y amazónica que es donde vislumbramos que se generarán la mayoría de los conflictos y tensiones entre los movimientos sociales, el Estado y las empresas multinacionales. Este cambio de viraje de las acciones colectivas de los movimientos sociales a partir de 2010, donde se vislumbran algunos cuestionamientos y enfrentamientos con el gobierno del MAS en torno a los territorios y los recursos naturales podrían implicar señales del nacimiento de un nuevo ciclo de protestas que ponga en crisis la legitimidad con al que contaba hasta ahora el gobierno de Evo Morales en el seno de los movimientos sociales bolivianos y en los sectores populares. Sin embargo, aun es muy difícil poder evaluar estas tendencias en términos de ciclos de protesta pues es necesaria una mayor perspectiva históricotemporal.

Las tensiones entre el modelo de desarrollo del Estado en Bolivia y los "mundos de vida" del pueblo Guaraní

Nos interesa problematizar la idea predominante en muchos análisis del proceso boliviano que sostiene que el gobierno de Evo Morales es un cristalizador del proceso de movilizaciones sociales de las últimas décadas, llevando a cabo desde el andamiaje estatal aquellas transformaciones enunciadas por los movimientos sociales bolivianos (Sader, 2008). También nos interesa problematizar la lectura contrapuesta que ve en el gobierno del MAS una claudicación plena de aquellas luchas y demandas en el marco de políticas reformistas que no influyen en cambios sustanciales en los núcleos de la dominación colonial y capitalista en Bolivia (Mamani, 2007). Por ello analizamos este proceso político y social desde una mirada que nos permita comprender al gobierno del MAS como un elemento más del complejo proceso de movilización social boliviano que no puede entenderse en los últimos años únicamente desde las políticas gubernamentales del MAS, pero tampoco puede comprenderse sin tenerlas en cuenta. Así, las tensiones entre movimientos sociales y Estado adquieren dimensiones cada vez más complejas y, en parte, es en la disputa por los territorios donde se dirimen o donde se vislumbran con mayor claridad estos procesos de diálogo, tensiones y conflictos.

Cuando el propio gobierno está impulsando la explotación de los recursos naturales, de gas, de otros recursos que existen en el país, entonces pareciera que para qué sirve que vamos a plantear que ya no se siga haciendo estas cosas que contaminan. Cuando el propio gobierno está firmando para que sigan explorando para que sigan explotando, impulsando que se perforen los pozos, entonces pareciera que es difícil decir basta, pero yo creo que estamos haciendo bien, todos los pueblos indígenas respecto a esto para que no siga contaminando. No se cómo lo tomará el Presidente, porque pareciera que se contradice él sólo. Nosotros como pueblo guaraní 
tenemos que plantearle esto, no para hacerle contra sino presentarle nuestra realidad como pueblo, para defender de verdad la Madre Tierra (Entrevista a Román Gómez, Mburuvicha Guasu de la APG Karaparí, 2010).

Nos resulta interesante señalar también que fue el gobierno del MAS el que recuperó en parte la institucionalidad política estatal que se encontraba en profunda crisis durante los primeros dos ciclos de protesta señalados. En este sentido, la relación del Estado con los movimientos sociales también se institucionalizó y, en esa relación, el Estado y también los propios movimientos sociales asumieron relaciones "corporativas" en torno a demandas sectoriales (Svampa, 2010), dejando de lado las facetas disruptivas en el marco de canalizar las demandas sectoriales de las organizaciones a través del andamiaje estatal tradicional (que incluyó la obtención de cargos para algunos dirigentes de los movimientos sociales), más algunas nuevas estructuras específicas que se propulsaron desde el gobierno del MAS como el Viceministerio de Tierras (encargado de la reforma agraria), o el Viceministerio de los Movimientos Sociales (encargado de atender las demandas específicas de los sindicatos y organizaciones populares urbanas, indígenas y campesinas).

En este sentido, siguiendo a Luis Tapia (2007: 150) vemos que el gobierno del MAS "es responsable de haber llevado a varias de estas organizaciones a su faceta más corporativa de sociedad civil, por lo tanto más conservadora (...) quitándole la dimensión de fuerza política que más bien plantea problemas generales y articula fuerza hacia reformas más globales en el país. Obviamente, otra parte de la responsabilidad está en las mismas organizaciones; y la explicación estaría en la historia de estas organizaciones en tanto instituciones de la sociedad civil, que las lleva a asumir, nuevamente, más esa faceta de corporación que de movimiento social". En efecto, el cambio de la relación no supone un movimiento unidireccional, sino que es el conjunto de los actores relacionados quienes han cambiado estas lógicas en las arenas de diálogo y conflicto, aunque la responsabilidad principal aparece entre quienes ejercen actualmente el poder estatal en Bolivia y que provienen de los propios movimientos sociales. La tensión entre la heteronomía estatal y la autonomía de los movimientos sociales se encuentra obviamente en la lógica política del Estado, pero también en el seno de los propios movimientos sociales que se encuentran tensionados entre el carácter disruptivo de lo político y social, y las demandas sectoriales y corporativas ligadas a la lógica estatal.

En el caso de la APG coexiste también esta mirada "neodesarrollista" con una mirada anclada en valores y mundos de vida inscritos en las tradiciones culturales del pueblo Guaraní, matizadas, traducidas y yuxtapuestas con la coyuntura política actual y con las formas políticas de la sociedad mestiza. En este sentido, los "mundos de vida" guaraní implican una forma "abigarrada" entre las tradiciones culturales y los "usos y costumbres" y lógicas políticas y sindicales aprendidas por las comunidades y sus dirigentes en estos años de articulación, además de acciones colectivas conjuntas con un vasto conglomerado de movimientos sociales indígenas, 
campesinos y urbanos que fueron permeando y transformando la identidad del pueblo guaraní. Sin embargo, uno de los núcleos del pensamiento político de los guaraní y de su vivencia política se encuentra anclado en diferentes nociones o conceptos ligados a sus tradiciones culturales y organizativas. Expondremos brevemente algunas de estas nociones como forma explicativa de las alternativas planteadas por la APG a la noción de desarrollo y al uso ilimitado de los recursos naturales: Nande Reko, Teko Kavi e Ivy Marai.

La noción del Ñande Reko es el pilar de la cultura y la identidad guaraní. Ñande significa "nuestro" y Reko denomina de manera amplia lo que en la cosmovisión occidental se considera "cultura". "El ÑandeReko significa mucho para nosotros, una cultura que compone todo, ¿no? La visión, la cosmovisión de cómo vivir bien, ¿no?” (Entrevista a Justino Zambrana, presidente del CCGTT de la APG Tarija, 2010).

En el sentido que le otorgan los guaraní, Nande Reko se traduce como "nuestro modo de ser y estar". De este modo, implica la cosmovisión, los aspectos religiosos, económicos, políticos y sociales del entramado comunitario de los guaraní. Así, aparece como proyección política y no sólo como una recuperación de las tradiciones ancestrales. Este concepto aparece incipientemente como alternativa política a la idea de "desarrollo" impuesta desde la matriz colonial/estatal/capitalista para los pueblos indígenas. Yuxtapone nociones ancestrales con las prácticas económicas, culturales y políticas actuales del pueblo guaraní.

Ya se ha pedido que se descarte definitivamente el concepto de desarrollo, el concepto desarrollista del mundo occidental, y que más bien se trata de estructurar esa concepción del “vivir bien”, del ÑandeReko, de lo que significa para el guaraní vivir bien. No solamente encasillado y enmarcado en esos idearios tradicionales, digamos, de "la tierra sin mal", la tierra del descanso, la paz, sino ya en un contexto actual y hacia el futuro. Cómo lograr mejorar condiciones materiales de vida sin perder, rescatando más bien, los valores de la identidad cultural guaraní (Entrevista a Hernán Ruiz, Equipo Técnico de la APG de Tarija, 2010).

La noción de Teko Kavi("buen vivir") se encuentra contenida en el Ñande Reko que actúa como la noción articuladora de la cosmovisión guaraní tradicional, pero también como proyecto político en la actualidad, en el marco de -por ejemplolos debates acerca de la construcción de un Estado plurinacional, las autonomías indígenas $\mathrm{y}$, por supuesto, la gestión y utilización de los territorios, los recursos naturales, especialmente los hidrocarburos. Finalmente, en torno a las disputas de los territorios podemos afirmar que el Ñande Reko, como "modo de ser", también 
implica un "modo de estar", una forma específica de habitar y practicar los territorios ligada a lógicas de reciprocidad con la naturaleza, con lógicas económicas alejadas de la idea de acumulación de bienes y de la acumulación de capital, sino que, como vimos anteriormente, se encuentran ligadas a lógicas productivas propias de los guaraní orientadas al autoconsumo y al intercambio de dones en el marco de pautas culturales que son definidas de manera colectiva por las propias comunidades.

Por último, otro concepto nodal entre la cosmovisión de los guaraní es el de Ivy Marai, que significa la "Tierra sin Mal”. Es una noción mítica que remite a un lugar-un espacio-tiempo- ideal para vivir en comunidad, donde pueda cosecharse buen maíz, donde haya animales, frutos, madera, miel y otras cosas necesarias para la reproducción de la vida de la comunidad.

La Tierra sin Mal para nosotros es un territorio, así digamos, virgen, que no tengan que entrar a cortar maderas para sacar a la venta, que no haiga ese destrozo, o sea que sea un bosque alto, un bosque así libre, digamos, donde haiga animales, donde haiga fruta, donde haiga miel para poder cosechar, o sea eso es un terreno sin mal, ¿por qué? Porque está sanito. Tiene animales, tiene fruta, tiene miel, tiene de todo para poder sustentarse ya, uno va, saca, cosecha cuando uno quiere y nadie dice nada (Entrevista a Aída Velázquez, Asambleísta Regional Gran Chaco, APG Yacuiba, 2010).

Esta idea mítica llevó a que los guaraní migraran históricamente hacia la búsqueda de la "Tierra sin Mal”, cuando alguna crisis económica, religiosa o política hacía insostenible la permanencia de una comunidad o parte de ella en un territorio determinado. En la actualidad esta noción se ha resignificado para remitir, junto con el Ñande Reko, al proyecto emancipatorio de los Guaraní.

La Tierra sin Mal es un sueño grande que el pueblo siempre ha soñado vivir bien, ¿no? Cuando hablamos de la Tierra sin Mal es que nadie sufra, ¿no? 0 sea, donde siempre el guaraní ha soñado ser, que no les falte ni comida, ni salud, que esté bien de salud, que esté bien de educación, que esté bien en tema de su territorio, que esté bien en tema de producción, en su infraestructura, en los recursos naturales, ¿no? Pensar que el pueblo esté unido, ¿no? Eso siempre se ha dicho, que el pueblo guaraní ha buscado la Tierra sin Mal, la unidad del pueblo y vivir bien. Que no nos falte nada, esa es la Tierra sin Mal. Y la Tierra sin Mal vamos a construir cuando el pueblo guaraní empiece ya a definir su territorio, cuando empiece ya a definir su 
proceso de autonomía, cuando empiece a definir su destino en base a su cosmovisión, ¿no? (Entrevista a Justino Zambrana, presidente del CCGTT de la APG Tarija, 2010).

Estos conceptos, que aparecen como resignificaciones construidas por los propios actores sociales en la reificación de sus "mundos de vida", permiten observar las contradicciones y tensiones entre una forma de uso y apropiación del territorio y los recursos naturales en reciprocidad con la naturaleza y el medio ambiente; y una lógica extractiva y de uso económico del territorio y los recursos naturales como mercancía, por más de que ésta apropiación sea distribuida de manera más o menos equitativa entre el conjunto de la población, tal como lo realiza en la actualidad el gobierno del MAS en Bolivia. De este modo, podemos platear que esta contradicción entre la APG y el pueblo guaraní, al igual que otros pueblos indígenas de Bolivia, marcan al incipiente ciclo de protesta que estaría emergiendo desde los movimientos sociales del país andino en los últimos años.

\section{Conclusiones}

En los ciclos de protesta planteados, la APG participó activamente de algunas de las movilizaciones de los indígenas de tierras bajas durante el primer ciclo de protesta señalado, aunque también participó durante el segundo ciclo de protesta en episodios de la "Guerra del Gas" y las movilizaciones posteriores, realizando bloqueos en los yacimientos hidrocarburíferos, principalmente en la zona del Itika Guasu. Los dos primeros ciclos de protesta le sirvieron de marco de oportunidades a la APG de Tarija para consolidar su proceso organizativo e ir creciendo en nuevas regiones del departamento. En efecto, desde el núcleo original del Itika Guasu la APG acrecentó su influencia hacia la zona del Gran Chaco, estableciendo nuevas capitanías generales en Villa Montes, Yacuiba y Karaparí. En los últimos años el proceso de organización del pueblo guaraní de Tarija se está expandiendo hacia la provincia de Bermejo en la frontera con la Argentina, en la región de Las Yungas, donde sin embargo aun no han logrado consolidarse capitanías regionales y más bien se trabaja en la recuperación de la identidad guaraní a nivel de comunidades. Durante el tercer ciclo de protestas, ahora bajo el gobierno de Evo Morales, la APG aparece como una de las organizaciones más dinámicas en las movilizaciones y acciones colectivas en defensa de sus territorios y los recursos naturales, ya sea dentro de las TCO's reconocidas como tales, así como en los territorios que habitan y resignifican en su práctica cotidiana las comunidades guaraní de Chuquisaca, Santa Cruz y Tarija.

Para los pueblos indígenas de Bolivia en general, y para el pueblo guaraní en particular, la demanda de territorio se encuentra asociada a la idea de autonomía. La obtención de reconocimiento territorial por parte del Estado, implica, desde la perspectiva de los guaraní, la obtención de la capacidad de autogestión de esos territorios y prácticas de autogobierno más o menos formales. Desde un discurso 
descolonial, la APG demanda la construcción de formas de autogobierno enraizadas en los "usos y costumbres" organizativos de los guaraní, así como e la posibilidad de decidir acerca del uso que se le da al territorio en cuestión y los recursos naturales que allí se encuentran.La cuestión de la autonomía indígena aparece como un horizonte concreto de las acciones colectivas de la APG, tanto en torno a la construcción cotidiana de "campos de experimentación social" (De Sousa Santos, 2003) en sus territorios ancestrales como también se puede vislumbrar en las demandas por el reconocimiento territorial y cultural, por la defensa de los recursos naturales y por la construcción de un Estado "plurinacional” y “descolonizado".

La movilización de los pueblos indígenas en Bolivia mantiene de manera latente la condición abigarrada de la sociedad, así las demandasde los guaraní en torno a la autonomía indígena y la autogestión de los territorios y los recursos naturales se inscriben en una lógica disruptiva del orden social vigente posicionados desde otra sociedad subalterna que impugna la sociedad dominante, "es la comunidad, con su sistema de relaciones sociales y su estructura de autoridades, la que se mueve contra la forma política dominante que ha subsumido de manera colonial la vida política de los pueblos dominados" (Tapia, 2008:71). Sin embargo, la construcción de la autonomía guaraní tiene diversas limitaciones, algunas son exógenas al proceso organizativo de la APG como por ejemplo las disputas territoriales con las empresas de hidrocarburos y la consiguiente dificultad en lograr el reconocimiento de sus territorios de manera integral o la injerencia de los partidos políticos del sistema institucional en la organización comunitaria y en la propia APG. Otras limitaciones aparecen en las dinámicas internas de la APG ya que el proceso organizativo del pueblo guaraní se encuentra aún en construcción. Este proceso presenta múltiples contradicciones en torno a los objetivos políticos, a las formas de gestión y apropiación de los territorios, a los modos de relacionamiento con el Estado y las empresas de hidrocarburos con quienes disputan los territorios.

Esta falta de consolidación del proceso organizativo en la búsqueda permanente de una nueva forma que recupere las formas tradicionales, aunque adaptadas a la sociedad abigarrada en la cual se encuentra inscripta, aparece como uno de los principales desafíos para construir la autonomía indígena desde las propias lógicas del pueblo guaraní. En el actual contexto político de Bolivia, emerge la posibilidad de que se habiliten estos procesos de autonomía indígena en el marco institucional de la construcción de un Estado plurinacional a nivel nacional. En este sentido, las disputas por los territorios ancestrales implican la posibilidad de una autogestión plena del pueblo guaraní en términos locales, pero también el desafío de construir una forma "descolonizada" de adscripción política de los pueblos indígenas en el contexto de un proyecto (pluri)nacional en Bolivia. En efecto, la construcción de un Estado plurinacional debe implicar el reconocimiento integral de los territorios indígenas, sus formas de autogobierno y de autogestión de los recursos naturales. De otra manera la conformación del "nuevo" Estado continuará manteniendo su matriz colonial. 


\section{Referencias Bibliográficas}

Albó, Xavier (1990).Los guaraní-chiriguan, 3. La comunidad hoy. La Paz: CIPCA.

Ceceña, Ana Esther (2005).La guerra por el agua y por la vida. Buenos Aires: Ediciones Madres de Plaza de Mayo.

Chávez, M., L. Choque, O. Olivera, P. Mamani, P. Chávez, R. Prada, R. Gutiérrez, R. Bautista, O. Vega, J. Viaña y L. Tapia (2006).Sujetos y formas de la transformación política en Bolivia. La Paz: Editorial Tercera Piel.

Chávez, Patricia, DuniaMokrani y Pilar Uriona (2010). “Una década de movimientos sociales en Boliva”. Observatorio Social de América Latina, $\mathrm{N}^{\circ} 28$, CLACSO, Buenos Aires.[pp. 71-93]

De Sousa Santos, Boaventura (2003).Crítica de la razón indolente: contra el desperdicio de la experiencia. Bilbao:Desclée de Brouwer.

De Sousa Santos, Boaventura(2010).Refundación del Estado en América Latina. Perspectivas desde una epistemologíadelSur. Buenos Aires: Antropofagia.

Fornillo, Bruno (2010). "Rupturas y dilemas de la Reforma Agraria durante la primera presidencia de Evo Morales”. En Juan Hernández, Marisa Armida y Augusto Bartolini (Comps.),Bolivia: Conflicto y cambio social[pp. 167-183]. Buenos Aires: Editorial NewenMapu.

García Linera, A., M. Chávez y P. Costas (2008).Sociología de los movimientos sociales. Estructuras de movilización, repertorios culturales y acción política. La Paz: Plural.

García Linera, A.,R. Gutiérrez,R. Prada y L. Tapia (2007).El retorno de la Bolivia plebeya. La Paz: Muela del Diablo. 
Hernández, Juan, Marisa Armida y Augusto Bartolini (Comps.) (2010).Bolivia: Conflicto y cambio social. Buenos Aires: Editorial NewenMapu.

Mamani, Pablo (Ed.) (2007). Evo Morales entre entornos blancoides, rearticulación de las oligarquías y movimientos indígenas, Willka, $N^{\circ} 1$, CADES, El Alto.

MançanoFernandes, Bernardo (2005). “Movimientos socio-territoriales y movimientos socio-espaciales”.Observatorio Social de América Latina, №16, CLACSO, Buenos Aires: [pp. 273-283].

Meliá, Bartolomeu (1988).Los guaraní-chiriguano 1. ÑandeReko nuestro modo de ser y bibliografía general comentada. La Paz: CIPCA.

Orozco Ramírez, S.,A. García Linera y P. Stefanoni (2006). “No somos juguete de nadie..." Análisis de la relación de movimientos sociales, recursos naturales, Estado y descentralización.Cochabamba: Plural Editores.

Orozco, Shirley y Jorge Viaña (2007). "El cierre de un ciclo y la compleja relación movimientos sociales-gobierno en Bolivia”. Observatorio Social de América Latina, $\mathrm{N}^{\circ}$ 22, CLACSO, Buenos Aires: [pp. 119-129].

Paz Patiño, Sarela (Coord.) (2004).Territorios Indígenas \& Empresas Petroleras. Cochabamba: CENDA.

Pifarré, Francisco (1989).Los guaraní-chiriguano 2. Historia de un Pueblo. La Paz: CIPCA.

Porto Gonçalves, Walter (2002). “Da geografia ás geo-grafías: um mundo em busca de novas territorialidades”.EnA. Ceceña y E. Sader (Coord.), La guerra infinita. Hegemonía y terror mundial[pp.217-256]. Buenos Aires: CLACSO.

Prada, Raúl (2006).Horizontes de la Asamblea Constituyente.La Paz: Yachaiwasi. 
Rivera Cusicanqui, Silvia (2003).Oprimidos pero no vencidos. Luchas del campesinado aymara y qwechwa de Bolivia, 1900-1980. La Paz:Aruwiyiri.

Svampa, M., P. Stefanoni y B. Fornillo (2010).Debatir Bolivia. Los contornos de un proyecto de descolonización. Buenos Aires: Taurus.

Svampa, Maristella y Pablo Stefanoni (Comp.) (2007).Bolivia: memoria, insurgencia y movimientos sociales. Buenos Aires: CLACSO-El Colectivo.

Tapia, Luis (2007). "Los movimientos sociales en la coyuntura del gobierno del MAS”.Willka, No 1, CADES, El Alto: [pp. 141-150].

Tapia, Luis (2008).Política Salvaje. Buenos Aires: Muela del Diablo-CLACSO.

Tarrow, Sidney (2009) .El poder en movimiento. Los movimientos sociales, la acción colectiva y la política. Madrid: Alianza.

Zavaleta Mercado, René (2008).Lo nacional popular en Bolivia. La Paz: Plural. 